\title{
The Primary Motor Cortex Stimulation Attenuates Cold Allodynia in a Chronic Peripheral Neuropathic Pain Condition in Rattus norvegicus
}

\author{
Priscila Medeiros ${ }^{1,2^{*}}$, Sylmara Esther Negrini-Ferrari1 ${ }^{*}$, Ana Carolina Medeiros ${ }^{1,2 *}$, \\ Lais Leite Ferreira ${ }^{3,4,5}$, Josie Resende Torres da Silva ${ }^{4,5}$, José Aparecido da Silva ${ }^{6,7}$, \\ Norberto Cysne Coimbra ${ }^{2,8}$, Renato Leonardo de Freitas 1,2,3,5,8\#
}

\author{
${ }^{1}$ Neurosciences aboratory of Pain \& Emotions and Multi-User Centre of Neuroelectrophysiology, \\ Department of Surgery and Anatomy, Ribeirão Preto Medical School of the University of São Paulo, Ribeirão Preto, São Paulo, Brazil \\ ${ }^{2}$ Laboratory of Neuroanatomy and Neuropsychobiology, Department of Pharmacology, Ribeirão Preto Medical School of the \\ University of São Paulo (FMRP-USP), Ribeirão Preto (SP), Brazil \\ ${ }^{3}$ Biomedical Sciences Institute, Federal University of Alfenas (UNIFAL-MG), Alfenas, Minas Gerais (MG), Brazil \\ ${ }^{4}$ Motricity Sciences Institute, UNIFAL-MG, Alfenas, MG, Brazil \\ ${ }^{5}$ Post-Graduate Program of Biosciences Applied to Health (PPGB), UNIFAL, Biomedical Sciences Institute, Alfenas, MG, Brazil \\ ${ }^{6}$ Department of Psychology, Ribeirão Preto School of Philosophy, Sciences and Literature of the University of São Paulo \\ FFCLRP-USP, Ribeirão Preto, Brazil \\ ${ }^{7}$ Department of Psychology, Federal University of Juiz de Fora (UFJF-MG), Juiz de Fora (MG), Brazil \\ ${ }^{8}$ Behavioural Neurosciences Institute (INeC), Ribeirão Preto (SP), Brazil \\ Email: "defreitas.rl@gmail.com
}

How to cite this paper: Medeiros, P., Negrini-Ferrari, S.E., Medeiros, A.C., Ferreira, L.L., da Silva, J.R.T., da Silva, J.A., Coimbra, N.C. and de Freitas, R.L. (2019) The Primary Motor Cortex Stimulation Attenuates Cold Allodynia in a Chronic Peripheral Neuropathic Pain Condition in Rattus norvegicus. World Journal of Neuroscience, 9, 138-152.

https://doi.org/10.4236/wjns.2019.93009

Received: April 12, 2019

Accepted: July 29, 2019

Published: August 1, 2019

Copyright $\odot 2019$ by author(s) and Scientific Research Publishing Inc. This work is licensed under the Creative Commons Attribution International License (CC BY 4.0).

\begin{abstract}
Background: The primary motor cortex $\left(\mathrm{M}_{1}\right)$ stimulation (MCS) is a useful tool for attenuation of the peripheral neuropathic pain in patients with pharmacologically refractory pain. Furthermore, that neurological procedure may also cause antinociception in rodents with neuropathic pain. Cold allodynia is a frequent clinical finding in patients with neuropathic pain, then, we evaluated if an adapted model of neuropathy induced by chronic constriction injury (CCI) of the ischiadicus nervus (sciatic nerve) produces cold allodynia in an animal model of chronic pain. In addition, we also investigated the effect of the electrical stimulation of the $\mathrm{M}_{1}$ on chronic neuropathic pain condition in laboratory animals. Methods: Male Wistar rats were used. An adapted model of peripheral mononeuropathy induced by CCI was carried out by placing a single loose ligature around the right sciatic nerve. The acetone test was used to evaluate the cold allodynia in CCI or Sham (without ligature) rats. The MCS $\left(\mathrm{M}_{1}\right)$ was performed at low-frequency $(20 \mu \mathrm{A}, 100 \mathrm{~Hz})$ during
\end{abstract} http://creativecommons.org/licenses/by/4.0/ 
$15 \mathrm{~s}$ by deep brain stimulation (DBS-Thomas Recording device) 21 days after CCI or Sham procedures. The cold allodynia was measured before and immediately after the neurostimulation of $\mathrm{M}_{1}$ in the following time-window: 0 , 15 and 30 min after MCS. Results: Cold allodynia threshold increased in animals with chronic neuropathic pain submitted to the acetone test 21 days after the CCI surgery. The $\mathrm{M}_{1}$-stimulation by DBS procedure decreased the cold allodynia immediately and until $30 \mathrm{~min}$ after $\mathrm{M}_{1}$-stimulation in rats with chronic neuropathic pain. Conclusion: The current proposal for a CCI model by a single loose ligature of the sciatic nerve can be employed as an experimental model of chronic neuropathic pain in rats submitted to peripheral nervous system injury. The $\mathrm{M}_{1}$-stimulation produced antinociception in rats with chronic neuropathic pain. Thus, we reinforced that the MCS decreases cold allodynia in laboratory animals submitted to persistent sciatic nerve constriction and can be a more reasonable procedure for the treatment of chronic intractable neuropathic pain.

\section{Keywords}

Peripheral Neuropathic Pain, Chronic Pain, Sciatic Nerve, Chronic Constriction Injury, Cold Allodynia, Primary Motor Cortex Stimulation, Antinociception

\section{Introduction}

The most recent definition of pain is given by Williams and Craig [1] in 2016, who say that "pain is a distressing experience associated with actual or potential tissue damage with the sensory, emotional, cognitive and social component". When such injury or dysfunction is related to the central nervous system activity, the pain is classified as neuropathic pain [2].

The incidence rate of chronic pain in the world is about 8\% [3] [4]; however, the prevalence of neuropathic pain is estimated to be between $1 \%$ and $23 \%$ [4]. The Institute of Medicine reported that 100 million Americans suffer from chronic pain at the cost of $\$ 600$ billion [5].

Recently, Carvalho and collaborators [6] reported high prevalence and severity of chronic pain and suggest that it is a public health problem in Brazil. In fact, there is a evidence that chronic pain affects more than two-thirds of the population of Brazil. Risk factors are being a woman, advanced age and low levels of household income. There is a need for improvement of health policies in Brazil for patients with chronic pain.

Pain can also be defined as acute when self-limited and caused by a specific disease or injury, or as chronic pain, considered a disease when it exceeds a normal cure time [7]. Neuropathic pain affects $5 \%$ of the Brazilian population [8] affecting the quality of life and reducing productivity [9]. Neuropathic pain is often severe, and it can afflict patients for their entire lifetime. 
Many studies have been performed to better understand the neurobiology of pain, considering its mechanisms, neural bases and comorbidities, as well as to find new forms of treatment. A novel target structure of these studies is the motor cortex (MC). Motor cortex stimulation (MCS) is a novel treatment modality used to modulate neuropathic pain by electrical stimulation of the brain [10]. That procedure was introduced by Tsubokawa et al. in 1991 [11] and has been used in clinical practice as an additional treatment of neuropathic pain [12] [13]. Since then, several studies have been done corroborating the pioneering results by Tsubokawa team [14] [15], although the neural bases of the analgesic mechanisms involved in that procedure have not been well established yet.

As a matter of fact, MCS is a neuromodulation therapy used for the treatment of neuropathic pain and it has shown good efficacy in the treatment of patients suffering from a pain disorder [14] [15]. Mixed results after MCS relieving pain in approximately $50 \%$ in patients with neuropathic pain were already demonstrated [13]. Based on that evidence, we evaluated the effect of MCS $\left(M_{1}\right)$ in rats with chronic neuropathic pain investigating the modulation of cold allodynia threshold by $\mathrm{M}_{1}$-stimulation in rats with chronic constriction injury (CCI) of the ischiadicus nervus (sciatic nerve).

The chronic and neuropathic pain present a heightened experience of pain caused by a noxious stimulus (hyperalgesia) and a stimulus that was innocuous becomes painful (allodynia) [16]. The prevalence of allodynia in neuropathic pain is likewise difficult to assess. In a questionnaire study recruiting more than 1600 patients with painful diabetic neuropathy [17], 18\% reported that light touching was painful, and $14 \%$ reported that cold or heat stimulus was occasionally painful. Only $47 \%$ with post-herpetic neuralgia had touch-evoked allodynia, although this is usually reported to be present in at least $70 \%$ of cases [18].

In another study involving 482 patients with different causes of neuropathic pain, 55\% had brush-evoked allodynia, whereas pain evoked by contact with cold objects was reported by $31 \%$ of patients, with pressure-evoked pain reported by $52 \%$ of patients. Any pain evoked by brush, pressure, or cold stimuli was present in $52 \%$ of patients with painful diabetic polyneuropathy and reported by $92 \%$ of patients with post-herpetic neuralgia [19].

The perception of innocuous and noxious cold is mediated by unmyelinated (C) and thinly myelinated (A $\delta$ ) fibres. Differential blocks of A fibres in human volunteers have shown that the sensitivity to innocuous cold is mediated by $A \delta$ fibres [20], although C fibres have also been shown to respond to innocuous cold [21] [22]. The existence of two types of neurons has been suggested: a low-threshold cool type, responding to activating temperatures close to $30^{\circ} \mathrm{C}$, and a high-threshold cold nociceptor neuron population, activated at temperatures less than $20^{\circ} \mathrm{C}$ [23].

Thus, the study of pain can be performed using chemical models, such as those using acetone, in which it is possible to evaluate the cold allodynia in animals that were submitted to an experimental procedure for the induction of neuropathy, such as the CCI, that was described by Bennet and Xie [24], and 
more recently, by Medeiros et al. (2019 submitted).

In this sense, the study of the role played by MCS in the modulation of the cold allodynia becomes interesting because there are no reports in the literature using MCS procedure for cold allodynia/hipernociception control in CCI rats. For this, in the present work, the effect of neurostimulation on the primary $\left(M_{1}\right)$ motor cortex (MC) in animals with chronic and neuropathic pain were submitted to the acetone test 21 days after chronic constriction injury (CCI) of the ischiadicus nervus.

\section{Material and Methods}

\subsection{Animals}

Male Wistar rats were housed four per cage with free access to food and water under controlled illumination (12:12 h light-dark cycle, light on 7am), temperature $\left(22^{\circ} \mathrm{C} \pm 1^{\circ} \mathrm{C}\right)$ and humidity $(55 \%-60 \%)$ for at least 1 week before the commencement of experiments in the animal care facility of the University of São Paulo (Campus of the University of São Paulo at Ribeirão Preto). All protocols were in compliance with the recommendations of the Committee for Ethics in Animal Experimentation (CETEA) of Ribeirão Preto Medical School of the University of São Paulo (FMRP-USP) (Process 015/2005 and 036/2017), which are in accordance with the Animal Research Ethics guidelines adopted by the National Council for Animal Experimentation Control (CONCEA) and with the International Association for the Study of Pain (IASP) guidelines for pain research on laboratory animals [25]. Experiments were conducted during the light phase of the cycle, and animals were randomly tested across that time-window. Each animal was used only in a single independent experimental group, and all efforts were made to minimise discomfort for the animals.

\subsection{Model of Neuropathic Pain: Chronic Constriction Injury (CCI) of the Ischiadicus Nervus}

The model used to induce experimental neuropathy was an adaptation of Bennet \& Xie's neuropathic pain (NP) model (1988). The aim of that procedure was lesioning the sciatic nerve by CCI procedure. Although the NP model proposed by Bennett and Xie consists of placing four loose ligatures around the sciatic nerve, we placed only one loose ligature around the right sciatic nerve using chromic catgut sutures. Our goal was to damage only one branch of the sciatic nerve by inducing swelling and then strangulation of that spinal nerve. Initially, the animals were anaesthetised through intramuscular (IM) administration of a solution consisting of $0.1 \mathrm{ml}$ of ketamine at $92 \mathrm{mg} / \mathrm{kg}$ (Ketamine ${ }^{\circledR}$; União Química Farmacêutica Nacional, São Paulo, Brazil) and $0.2 \mathrm{ml}$ of xylazine at $9.2 \mathrm{mg} / \mathrm{kg}$ (Dopaser ${ }^{\circledR}$; União Química Farmacêutica Nacional, São Paulo, Brazil). The right hind paw was shaved, and the skin was disinfected with povidone-iodine. Subsequently, a 15-mm longitudinal incision was made in the dorsolateral region of the thigh, at the level of the femur trochanter, on the right posterior limb. The 
sciatic nerve was exposed through dissection of the gluteus maximus muscle and biceps femoris, and a single ligature of 4 - 0 catgut thread was placed around the nerve, in contrast to the procedure described by Bennett \& Xie (1988). Finally, the skin incision was sutured with 5 - 0 mononylon thread. Sham surgery was performed by exposing the sciatic nerve as described above, without nerve ligation. The animals were then transferred to their home cages and left to recover. In our study, the rats were examined daily after the surgery and about weekly until 21 days. During this inspection, each animal was placed upon a table and carefully observed the condition of the affected hind paw (Figure 1).

\subsection{Cold Allodynia-Acetone Test}

The acetone test, conducted in an independent group of rodents, was used to measure cold allodynia. Each animal was placed in a transparent acrylic cubicle $(22 \times 16.5 \times 14 \mathrm{~cm})$ with a mesh floor. The rats $(\mathrm{n}=8)$ were habituated to the testing environment for at least $10 \mathrm{~min}$. Then, a drop of acetone $(50 \mu \mathrm{L})$ was injected into the centre of the plantar surface of the right hind paw through a glass syringe equipped with a blunted needle. The time the animal spent flicking or licking the stimulated paw during a $1 \mathrm{~min}$ period was recorded.
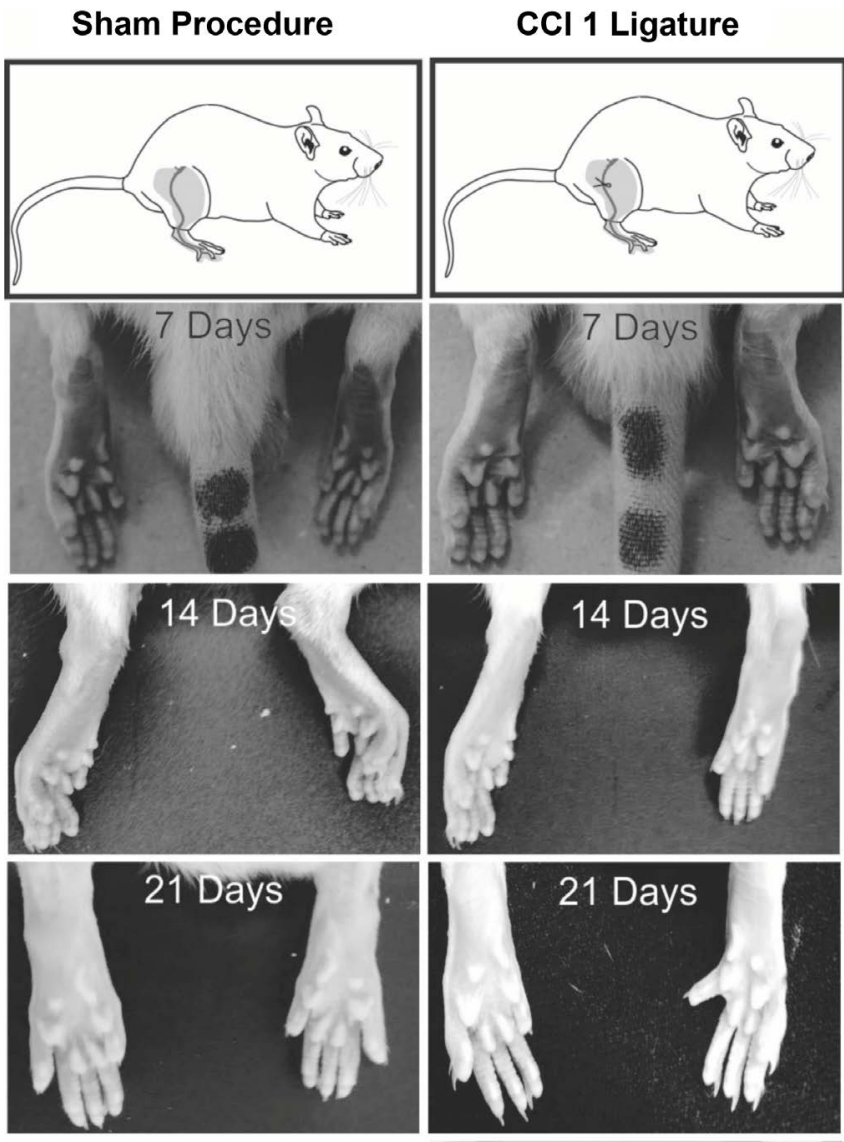

Figure 1. Illustration of the evolution of right paws of rats after surgery to induce sciatic nerve chronic constriction injury (CCI) and sham procedure recorded 7, 14 and 21 days after each procedure. 


\subsection{Stereotaxic Surgery}

An electrode was implanted in the primary $\left(M_{1}\right)$ motor cortex of rats. Fourteen days after either the Sham procedure or the CCI surgery for chronic neuropathy induction, the animals were anaesthetised with $92 \mathrm{mg} / \mathrm{kg}$ ketamine (Ketamine) and $9.2 \mathrm{mg} / \mathrm{kg}$ xylazine (Dopaser) and fixed in a stereotaxic frame (David Kopf, USA). The upper incisor bar was set at $3.3 \mathrm{~mm}$ below the interaural line such that the skull was horizontal between bregma and lambda. Subsequently, the electrode was fixed to the skull with acrylic resin and two stainless steel screws above the $\mathrm{M}_{1}$ (anteroposterior $=-1.1 \mathrm{~mm}$; mediolateral $=+1.5$; and dorsoventral $=-1.5 \mathrm{~mm}$ from the skull) in accordance with Paxinos and Watson's rat brain in stereotaxic coordinates atlas [26].

\subsection{Primary $\left(M_{1}\right)$ Motor Cortex Stimulation (MCS)}

Three weeks after the CCI or Sham surgeries and one week after stereotaxic surgery, it was performed the baseline 2 of acetone test in the animals. After that procedure, the animals were placed in a circular arena $(60 \mathrm{~cm}$ in diameter and 50 $\mathrm{cm}$ high with the floor divided into 12 sections) with the experimental compartment illuminated with a $40-\mathrm{W}$ fluorescent lamp (350 lx at the arena floor level). The animals were allowed a $3 \mathrm{~min}$ period of habituation. Afterwards, the electrode implanted in the midbrain was connected to a stimulus generator (STG3008-FA, Multichannel Systems, Germany) which allowed to apply current pulses (cathode pulse width $100 \mu$ s, pulse interval $100 \mu$ s and anode pulse width $100 \mu \mathrm{s}$, repeated during $15 \mathrm{~s}$ ). The $\mathrm{M}_{1}$ stimulation was made during $15 \mathrm{~s}$ at 20 $\mu \mathrm{A}$, immediately followed by acetone test for $30 \mathrm{~min}$ in the right paw. Each animal was used only once and received only one MCS. The temporal window of the experimental procedure was shown in Figure 2.

\subsection{Histological Analysis}

After testing, the rats were anaesthetised as previously described and perfused through the left cardiac ventricle with cold, oxygen-enriched, $\mathrm{Ca}^{++}$-free Tyrode's buffer $\left(40 \mathrm{~mL}\right.$ at $\left.4^{\circ} \mathrm{C}\right)$ and ice-cold paraformaldehyde [200 $\mathrm{mL}, 4 \%(\mathrm{w} / \mathrm{v})$ in 0.1 $\mathrm{M}$ sodium phosphate buffer, $\mathrm{pH}$ 7.3) for $15 \mathrm{~min}$ at a pressure of $50 \mathrm{mmHg}$ with a

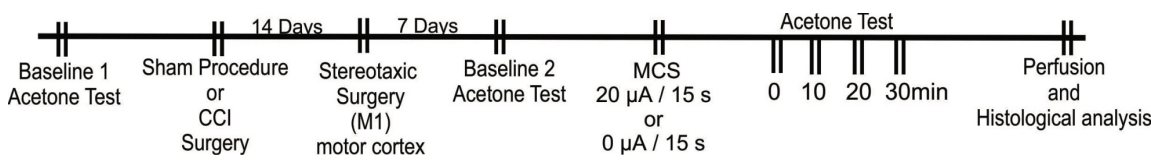

Figure 2. Timeline of the experimental procedure. The animals ( $n=8$ per group) were divided in different groups. The duration of cold stimulus-induced shakes and liking responses was measured once before the chronic constriction injury (CCI) or sham procedures (day 1 ), in the $14^{\text {th }}$ day the stereotaxic surgery was done for implantation of the electrical stimulation electrode. In the $21^{\text {st }}$ day after sciatic nerve CCI or sham procedures, the cold stimulus response was measured before the electrical stimulation of the primary $\left(M_{1}\right)$ motor cortex. The duration of shakes and liking was measured for $30 \mathrm{~min}$ after the cortical electrical stimulation. Subsequently, the animals were perfused and histological analyses were conducted. 
perfusion pump (Master Flex ${ }^{\circledR} \mathrm{L} / \mathrm{S}^{\mathrm{TM}}$ peristaltic tubing pump, East Bunker Court Vernon Hills, Illinois, USA). The brainstem was quickly sectioned, removed and immersed in fresh fixative for $4 \mathrm{~h}$ at $4^{\circ} \mathrm{C}$. It was then rinsed in $10 \%$ and $20 \% \mathrm{su}$ crose dissolved in $0.1 \mathrm{M}$ sodium phosphate buffer $(\mathrm{pH} 7.4)$ at $4^{\circ} \mathrm{C}$, for at least 12 $\mathrm{h}$ each solution. The central nervous system tissue pieces were immersed in 2-methylbutane (Sigma-Aldrich, St. Louis, Missouri, USA), frozen on dry ice, embedded in Tissue-Tek O.C.T., and cut with a cryostat (CM 1950 Leica, Wetzlar, Germany) at $-22^{\circ} \mathrm{C}$. Brain slices were subsequently mounted on glass slides (coated with chrome alum gelatin to prevent detachment) and stained with haematoxylin-eosin by using an Autostainer (CV 5030 Autostainer XL, Leica, Wetzlar, Germany). The positions of the guide cannula tips were defined according to the Paxinos and Watson atlas (2007) [25] under a motorised photomicroscope (AxioImager Z1; Zeiss, Oberkochen, Germany). Statistical analysis was performed exclusively with data from the animals in which evidence showed that the microinjection into the primary $\left(\mathrm{M}_{1}\right)$ motor cortex had been successfully carried out.

\subsection{Statistical Analysis}

The data are expressed as the mean \pm standard error of the mean (S.E.M.). Data regarding the cold allodynia threshold were subjected to a two-way repeated measures analysis of variance (split-plot ANOVA), followed, when appropriate, by Tukey's post hoc test. The procedure (electrical stimulation of $M_{1}$ ) was considered the independent factor, and the time was considered the dependent factor. Repeated measures ANOVA uses the same conceptual framework as classical ANOVA followed by Tukey's post hoc test [27] [28].

\subsection{Experimental Design}

Primary motor cortex stimulation (MCS) and the effect of that procedure on cold allodynia and chronic neuropathic pain induced by CCI.

The following experimental procedures (Figure 2) were performed in independent groups of animals:

- Neurophysiological study: 1-non-stimulation/Sham procedure rats $(\mathrm{n}=8)$, $2-\mathrm{MCS} /$ Sham procedure rats $(\mathrm{n}=8), 3-$ non-stimulation/CCI rats $(\mathrm{n}=8)$, and $4-\mathrm{MCS} / \mathrm{CCI}$ rats $(\mathrm{n}=8)$.

1) The animals had their basal nociceptive thresholds (baseline 1) measured through the acetone test; on the same day, each animal was anaesthetised and subjected to either a CCI or sham procedure;

2) Fourteen days after the $\mathrm{CCI}$ and sham procedures, the animals were anaesthetised and subjected to stereotaxic surgery for implantation of an electrode in the $\mathrm{M}_{1}$;

3) Seven days after the stereotaxic surgery and 21 days after the CCI or sham procedure, the nociceptive threshold of cold allodynia was measured by the acetone test (baseline 2) for each rat; 
4) Immediately after baseline 2 , the rats were connected to the neurostimulator (DBS machine) for MCS (electric current intensity: $20 \mu \mathrm{A}$; duration: $15 \mathrm{~s}$ ) in the open field apparatus, and after $1 \mathrm{~min}$, the rats were subjected to cold allodynia threshold recordings at 0,15 , and 30 min after MCS;

5) Twenty-four hours after the experiment, each rat was anaesthetised and perfused for the histochemical procedure.

\section{Results}

The primary motor cortex $\left(\mathrm{M}_{1}\right)$ electrical stimulation (MCS) attenuates the neuropathic pain (NP)

Histologically confirmed electrode tips situated in the primary $\left(M_{1}\right)$ motor cortex in CCI or Sham rats are shown in Figure 3.

To evaluate the involvement of $\mathrm{M}_{1}$ neurons during neuropathic pain induced by an adapted model of CCI, we stimulated the $M_{1}$ cortex 21 days after the CCI or sham procedure (right hind paw).

According to repeated measures Two-way ANOVA, there were statistically significant effects of treatment $\left[\mathrm{F}_{(3,21)}=27.39 ; \mathrm{p}<0.0001\right]$, of time $\left[\mathrm{F}_{(4,28)}=10.64\right.$; $\mathrm{p}<0.0001]$ and of treatment-by-time interaction $\left[\mathrm{F}_{(12,84)}=9.197 ; \mathrm{p}<0.0001\right]$, considering cold allodynia recorded 21 days after CCI induction (Figure 4). There was a significant decreased in cold allodynia in the neuropathic pain animals after the motor cortex stimulation at $20 \mu \mathrm{A}$ for 15 seconds compared with the neuropathic animals that did not receive stimulation for $30 \mathrm{~min}$ after deep brain stimulation (Tukey's post hock test; $\mathrm{P}<0.05$ ), as shown in Figure 4.

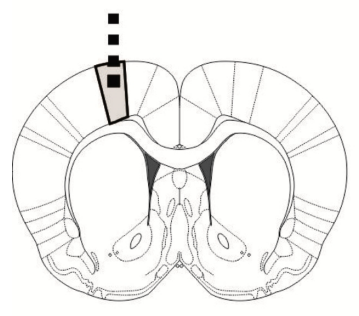

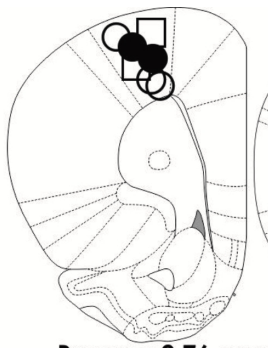

Bregma $2.76 \mathrm{~mm}$

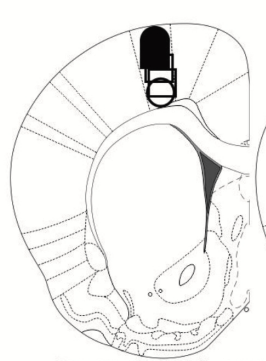

Bregma $1.44 \mathrm{~mm}$

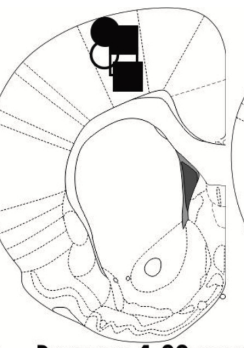

Bregma $1.80 \mathrm{~mm}$

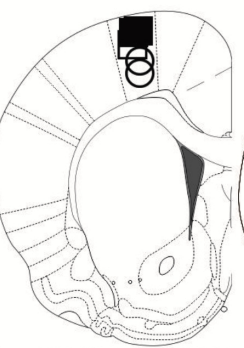

Bregma $1.28 \mathrm{~mm}$

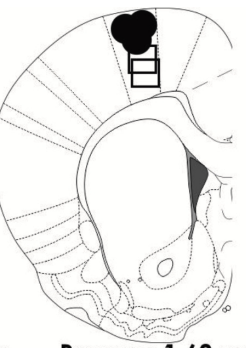

Bregma $1.68 \mathrm{~mm}$

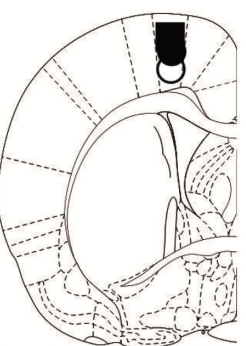

Bregma $-0.25 \mathrm{~mm}$

Figure 3. Diagrammatic representation of histological sites of the microelectrode implanted in the primary $\left(\mathrm{M}_{1}\right)$ motor cortex in accordance to Paxinos and Watson's stereotaxic atlas (2007). Motor cortex stimulation (MCS) at $0 \mu \mathrm{A} / 15 \mathrm{~s}-$ Sham group (o), MCS at $0 \mu \mathrm{A} / 15 \mathrm{~s}-$ chronic constriction injury $(\mathrm{CCI})$ of the right sciatic nerve group $(\bullet)$, MCS at $20 \mu \mathrm{A} / 15 \mathrm{~s}-$ Sham group (口), MCS at $20 \mu \mathrm{A} / 15 \mathrm{~s}-\mathrm{CCI}$ group (•). 


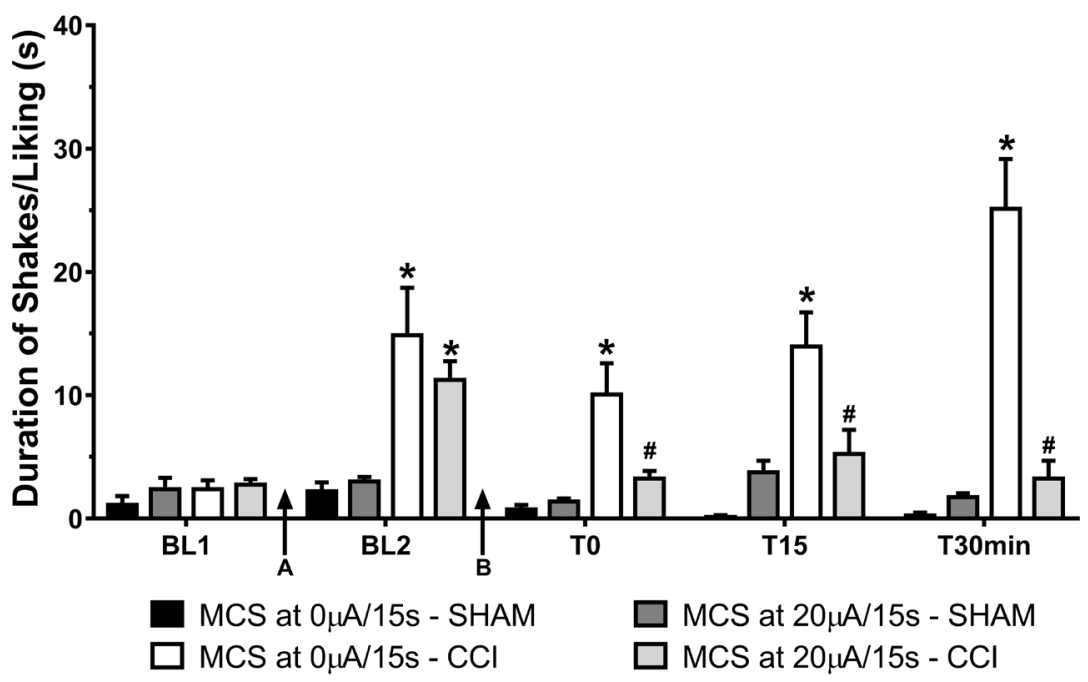

Figure 4. Effect of electrical stimulation of the primary motor $\left(M_{1}\right)$ cortex (MCS) on cold allodynia recorded in right hind paw of Wistar rats submitted to the neuropathic pain caused by chronic constriction injury (CCI) of the right sciatic nerve or sham procedure. BL1: baseline before the procedures. Arrow A: CCI or sham procedures. BL2: New baseline recorded at 21 days after the sham or CCI procedures. Arrow B: $\mathrm{M}_{1}$ cortex electrical stimulation (MCS) at $20 \mu \mathrm{A}$ or $0 \mu \mathrm{A} / 15 \mathrm{sec}$ after the BL2 recording in sham or CCI rats, followed by von Frey's test for $30 \mathrm{~min}$ after the intracortical stimulation. " $¥$ ” indicates significant differences $(\mathrm{P}<0.05)$ compared to the sham procedure, "\#” indicates significant differences $(\mathrm{P}<0.05)$ compared eMCS at $0 \mu \mathrm{A} / 15 \mathrm{~s}-\mathrm{CCI}$ group, according to two-way ANOVA followed by Tukey's post hoc test.

\section{Discussion}

In the present study, it was demonstrated that the electrical MCS on the $M_{1}$ decreased cold allodynia assessment by acetone test in Wistar rats. The nociceptive measured evaluating cold allodynia was made 21 days after CCI in animals with chronic neuropathic pain. These results suggest an antinociceptive effect caused by motor cerebral cortex electric stimulation (see graphical abstract in Figure 5).

According to Calcutt et al., 1997 [29], the neural mechanism of the thermal stimulation is not completely known, since the studies performed until now were focused only on a cerebral cortex stimulation. Interestingly, we showed here that the deep brain stimulation of the primary motor cortex reduced the cold thermal stimulus-related hyperalgesia. Considering the relevance of glutamatergic projections from the cerebral cortex [27] [30], these telencephalic fugal projections have a putative involvement in the neurochemical mechanisms of motor cortex stimulation-induced antinociception. Indeed, there are reports highlighting the relevance of N-methyl-D-aspartic acid receptor in the transcranial magnetic stimulation-induced analgesia [31].

Considering the neuropathic pain mechanisms studied in the present experimental model of chronic pain, peripheral lesion of spinal nerves leads the individual to an altered perception of innocuous stimuli and noxious stimuli, and therefore, pain is often felt in the limb at the touch, as demonstrated by the inability to detect a fine probe applied to the skin [32], and the information resulting 


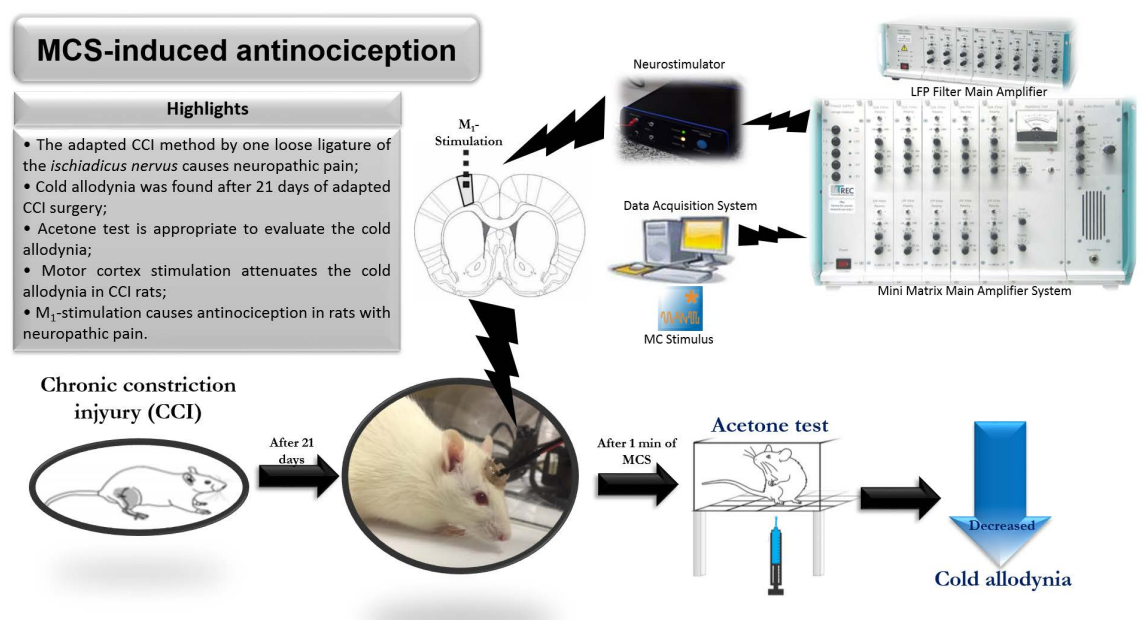

Figure 5. Graphical abstract: Motor cortex stimulation (MCS)-induced antinociception. Twenty-one days after chronic constriction injury (CCI) of the ischiadicus nervus (sciatic nerve), there was an increase in the cold allodynia threshold measured by the acetone test. The primary $\left(\mathrm{M}_{1}\right)$ MCS at $20 \mu \mathrm{A}$ during 20 seconds by Thomas Recording device (MC Stimulus software) decreased the cold allodynia in Wistar rats with chronic and neuropathic pain induced by CCI.

from the somatosensory cortex is altered. We might consider that the motor cortex stimulation causes a reorganisation of the sensory somatic information in the cerebral somatosensory cortex through a direct cortico-cortical functional brain connectivity, or through an indirect cortico-neostriatum-pallido-thalamocortical projection [33] Cortico-cortical neurons in superficial layers of motor cortex work with motor and sensory signals and might mediate sensorimotor integration [34].

Interestingly, there is evidence that tactile stimuli can be modulated by thalamus-efferent connections to reach both motor and somatosensory neocortical areas [35] [36]. The influence exerted by neocortical somatosensory areas on the motor cortex function is already known, and sensorimotor connexions are critical for efficient movements control, considering that the somatosensory connectivity has been shown to play relevant effects on the execution of motor plans [37]. Impairments of the connectivity between the motor and the somatosensory cortexes cause dexterity disfunctions [38]. Finally, reciprocal connections between the somatomotor cortical areas were already demonstrated in rodents [39]. Differences in synaptic function suggest specialisations within the sensorimotor connexions that may allow updating of sensory-motor inputs integration within the neocortex in response to changes in the sensory periphery [40].

Therefore, the acetone test procedure in animals with neuropathic pain was planned to evaluate sensitivity of low temperature-related ascending spino-thalamic pathways, and allows us to verify that there were decreases in the nociceptive threshold in these animals with chronic neurogenic mononeuropathy. These results corroborate studies performed in human beings, and considering that in the medical clinics, it is reported that the sensitivity for cold stimulus in patients 
with chronic neuropathic pain also showed increased [17] [18] [19].

It is known that the sensory dysfunction detected in patients with chronic neuropathic pain involves both mechanical and thermal dysfunctions and, as shown in the present study, rodents with neuropathic pain present markedly cold allodynia. Some studies using fRMI approaches before and after the injury demonstrated that the mechanical stimulation of the skin produced increased pain in the area of secondary hyperalgesia leading to greater activation in areas of the brain associated with pain signalling [41] [42].

In conclusion, the electrical stimulation of the primary motor cortical area caused analgesia in laboratory animals with neuropathic pain induced by CCI. Although additional studies are required to understand the relationship between MCS and neuropathic pain control, we might consider that the MCS acts not only as a cortico-cortical neuromodulator, but also as a putative descending modulator of pain pathways.

Further investigations of the neuroanatomical and neurochemical mechanisms involved in the anti-hypernociceptive phenomenon studied in the present work may contribute to the improvement of the clinical treatment of pharmacologically refractory chronic pain and neuropathy.

\section{Acknowledgements}

This research was supported by Fundação de Amparo à Pesquisa do Estado de São Paulo (FAPESP) (Research grant 2013/12916-0 and Multi-user Equipment grant 2014/11869-0). Neither of these funding sources had any role in the study design; in the collection, analysis, and interpretation of data, in the writing of the report, or in the decision to submit the paper for publication. Renato Leonardo de Freitas was supported by FAPESP (Scientific Initiation Scholarship grant 2001/03752-6, M.Sc. fellowship grant 2003/05256-1, post-doctoral fellowship grant 2009/17258-5, and researcher fellowship grant 2014/07902-2) and CAPES (Sc.D. fellowship grant). FAPESP also supported Priscila de Medeiros (Sc.D. fellowship grant 2012/25167-2; post-doctoral fellowship grant 2017/13560-5), Ana Carolina Medeiros (Scientific Initiation Scholarship grant FAPESP 2017/12116-4). The authors thank Daoud Hibrahim Elias-Filho, Paulo Castilho, Ariana Santana and Maria Rossatto for their expert technical assistance.

\section{Conflicts of Interest}

The authors declare that they have no conflicts of interest concerning the work presented herein.

\section{References}

[1] de Williams, A.C. and Craig, K.D. (2016) Updating the Definition of Pain. Pain, 157, 2420-2423. https://doi.org/10.1097/j.pain.0000000000000613

[2] Mann, J.J., Currier, D., Quiroz, J.A. and Manji, H.K. (2012) Neurobiology of Severe Mood and Anxiety Disorders. In: Brady, S.T., Siegel, G.J., Albers, R.W. and Price, D.L., Eds., Basic Neurochemistry, Academic Press, Cambridge, MA, 1021-1036. 
[3] Elliott, A.M., Smith, B.H., Penny, K.I., Smith, W.C. and Chambers, W.A. (1999) The Epidemiology of Chronic Pain in the Community. The Lancet, 354, 1248-1252. https://doi.org/10.1016/S0140-6736(99)03057-3

[4] Velly, A.M. and Mohit, S. (2018) Epidemiology of Pain and Relation to Psychiatric Disorders. Progress in Neuro-Psychopharmacology and Biological Psychiatry, 87, 159-167. https://doi.org/10.1016/j.pnpbp.2017.05.012

[5] Gaskin, D.J. and Richard, P. (2012) The Economic Costs of Pain in the United States. The Journal of Pain, 13, 715-724. https://doi.org/10.1016/j.jpain.2012.03.009

[6] de Carvalho, R.C., Maglioni, C.B., Machado, G.B., Araújo, J.E., Silva, J.R.T. and Silva, M.L. (2018) Prevalence and Characteristics of Chronic Pain in Brazil : A National Internet-Based Survey Study. Brazilian Journal of Pain, 1, 1-8. https://doi.org/10.5935/2595-0118.20180063

[7] Grichnik, K.P. and Ferrante, F.M. (1991) The Difference between Acute and Chronic Pain. Mount Sinai Journal of Medicine, 58, 217-220.

[8] de Souza, J.B., Grossmann, E., Perissinotti, D.M.N., de Oliveira Junior, J.O., da Fonseca, P.R.B. and Posso, I.D.P. (2017) Prevalence of Chronic Pain, Treatments, Perception, and Interference on Life Activities: Brazilian Population-Based Survey. Pain Research and Management, 2017, Article ID: 4643830. https://doi.org/10.1155/2017/4643830

[9] Navarro, A., Saldaña, M.T., Pérez, C., Masramón, X. and Rejas, J. (2012) Costs Health Resources Utilization Following Switching to Pregabalin in Individuals with Gabapentin-Refractory Neuropathic Pain: A Post Hoc Analysis. Pain Practice, 12, 382-393. https://doi.org/10.1111/j.1533-2500.2011.00515.x

[10] Lazorthes, Y., Sol, J.C., Fowo, S., Roux, F.E. and Verdié, J.C. (2007) Motor Cortex Stimulation for Neuropathic Pain. In: Sakas, D.E. and Simpson, B.A., Eds., Operative Neuromodulation. Acta Neurochirurgica Supplements, Springer, Vienna, 37-44. https://doi.org/10.1007/978-3-211-33081-4_4

[11] Tsubokawa, T., Katayama, Y., Yamamoto, T., Hirayama, T. and Koyama, S. (1991) Chronic Motor Cortex Stimulation for the Treatment of Central Pain. In: Hitchcock, E.R., Broggi, G., Burzaco, J., Martin-Rodriguez, J., Meyerson, B.A. and Tóth, S., Eds., Advances in Stereotactic and Functional Neurosurgery 9. Acta Neurochirurgica Supplementum, Springer, Vienna, 137-139. https://doi.org/10.1007/978-3-7091-9160-6_37

[12] Buchanan, R.J., Darrow, D., Monsivais, D., Nadasdy, Z. and Gjini, K. (2014) Motor Cortex Stimulation for Neuropathic Pain Syndromes: A Case Series Experience. NeuroReport, 25, 715-717. https://doi.org/10.1097/WNR.0000000000000174

[13] Nguyen, J.P., Keravel, Y., Feve, A., Uchiyama, T., Cesaro, P. and Le Guerinel, C. (1997) Treatment of Deafferentation Pain by Chronic Stimulation of the Motor Cortex: Report of a Series of 20 Cases. In: Ostertag, C.B., Thomas, D.G.T., Bosch, A., Linderoth, B. and Broggi, G., Eds., Advances in Stereotactic and Functional Neurosurgery 12. Acta Neurochirurgica Supplements, Springer, Vienna, 54-60. https://doi.org/10.1007/978-3-7091-6513-3_10

[14] Monsalve, G. (2012) Motor Cortex Stimulation for Facial Chronic Neuropathic Pain: A Review of the Literature. Surgical Neurology International, 3, S290-S311. https://doi.org/10.4103/2152-7806.103023

[15] Garcia-Larrea, L. and Peyron, R. (2007) Motor Cortex Stimulation for Neuropathic Pain: From Phenomenology to Mechanisms. NeuroImage, 37, S71-S79. https://doi.org/10.1016/j.neuroimage.2007.05.062

[16] Attal, N., Jazat, F., Kayser, V. and Guilbaud, G. (1990) Further Evidence for 
Pain-Related Behaviours in a Model of Unilateral Peripheral Mononeuropathy. Pain, 41, 235-251. https://doi.org/10.1016/0304-3959(90)90022-6

[17] Baron, R., Tölle, T.R., Gockel, U., Brosz, M. and Freynhagen, R. (2009) A Cross-Sectional Cohort Survey in 2100 Patients with Painful Diabetic Neuropathy and Postherpetic Neuralgia: Differences in Demographic Data and Sensory Symptoms. Pain, 146, 34-40. https://doi.org/10.1016/j.pain.2009.06.001

[18] Johnson, R.W., Wasner, G., Saddier, P. and Baron, R. (2008) Herpes Zoster and Postherpetic Neuralgia: Optimizing Management in the Elderly Patient. Drugs \& Aging, 25, 991-1006. https://doi.org/10.2165/0002512-200825120-00002

[19] Attal, N., Fermanian, C., Fermanian, J., Lanteri-Minet, M., Alchaar, H. and Bouhassira, D. (2008) Neuropathic Pain: Are There Distinct Subtypes Depending on the Aetiology or Anatomical Lesion? Pain, 138, 343-353. https://doi.org/10.1016/j.pain.2008.01.006

[20] Torebjörk, H.E. and Hallin, R.G. (1973) Perceptual Changes Accompanying Controlled Preferential Blocking of A and C Fibre Responses in Intact Human Skin Nerves. Experimental Brain Research, 16, 321-332. https://doi.org/10.1007/BF00233334

[21] Campero, M., Serra, J., Bostock, H. and Ochoa, J.L. (2001) Slowly Conducting Afferents Activated by Innocuous Low Temperature in Human Skin. The Journal of Physiology, 535, 855-865. https://doi.org/10.1111/j.1469-7793.2001.t01-1-00855.x

[22] Campero, M. and Bostock, H. (2010) Unmyelinated Afferents in Human Skin and Their Responsiveness to Low Temperature. Neuroscience Letters, 470, 188-192. https://doi.org/10.1016/j.neulet.2009.06.089

[23] Madrid, R., de la Peña, E., Donovan-Rodriguez, T., Belmonte, C. and Viana, F. (2009) Variable Threshold of Trigeminal Cold-Thermosensitive Neurons Is Determined by a Balance between TRPM8 and Kv1 Potassium Channels. Journal of Neuroscience, 29, 3120-3131. https://doi.org/10.1523/JNEUROSCI.4778-08.2009

[24] Bennett, G.J. and Xie, Y.K. (1988) A Peripheral Mononeuropathy in Rat that Produces Disorders of Pain Sensation Like Those Seen in Man. Pain, 33, 87-107. https://doi.org/10.1016/0304-3959(88)90209-6

[25] Zimmermann, M. (1983) Ethical Guidelines for Investigations of Experimental Pain in Conscious Animals. Pain, 16, 109-110. https://doi.org/10.1016/0304-3959(83)90201-4

[26] Paxinos, G. and Watson, C. (2007) The Rat Brain in Stereotaxic Coordinates. Academic Press, San Diego, 6.

[27] de Freitas, R.L., Salgado-Rohner, C.J., Biagioni, A.F., et al. (2014) NMDA and AMPA/Kainate Glutamatergic Receptors in the Prelimbic Medial Prefrontal Cortex Modulate the Elaborated Defensive Behavior and Innate Fear-Induced Antinociception Elicited by $\mathrm{GABA}_{\mathrm{A}}$ Receptor Blockade in the Medial Hypothalamus. Cerebral Cortex, 24, 1518-1528. https://doi.org/10.1093/cercor/bht001

[28] de Freitas, R.L., Medeiros, P., Khan, A.U. and Coimbra, N.C. (2016) $\mu_{1}$-Opioid Receptors in the Dorsomedial and Ventrolateral Columns of the Periaqueductal Grey Matter Are Critical for the Enhancement of Post-Ictal Antinociception. Synapse, 70, 519-530. https://doi.org/10.1002/syn.21926

[29] Calcutt, N.A. and Chaplan, S.R. (1977) Spinal Pharmacology of Tactile Allodynia in Diabetic Rats. British Journal of Pharmacology, 122, 1478-1482.

https://doi.org/10.1038/sj.bjp.0701538

[30] Falconi-Sobrinho, L.L., Dos Anjos-Garcia, T., Elias-Filho, D.H. and Coimbra, N.C. 
(2017) Unravelling Cortico-Hypothalamic Pathways Regulating Unconditioned Fear-Induced Antinociception and Defensive Behaviours. Neuropharmacology, 113, 367-385. https://doi.org/10.1016/j.neuropharm.2016.10.001

[31] Ciampi de Andrade, D., Mhalla, A., Adam, F., Texeira, M.J. and Bouhassira, D. (2014) Repetitive Transcranial Magnetic Stimulation Induced Analgesia Depends on N-Methyl-d-Aspartate Glutamate Receptors. Pain, 155, 598-605. https://doi.org/10.1016/j.pain.2013.12.022

[32] Campbell, J.N. and Meyer, R.A. (2006) Mechanism of Neuropathic Pain. Neuron, 52, 77-92. https://doi.org/10.1016/j.neuron.2006.09.021

[33] Cummiford, C.M., Nascimento, T.D., Foerster, B.R., Clauw, D.J., Zubieta, J. and Harris, R.E. (2016) Changes in Resting State Functional Connectivity after Repetitive Transcranial Direct Current Stimulation Applied to Motor Cortex in Fibromyalgia Patients. Arthritis Research \& Therapy, 18, 40. https://doi.org/10.1186/s13075-016-0934-0

[34] Mao, T., Kusefoglu, D., Hooks, B.M., Huber, D., Petreanu, L. and Svoboda, K. (2011) Long-Range Neuronal Circuits Underlying the Interaction between Sensory and Motor Cortex. Neuron, 72, 111-123. https://doi.org/10.1016/j.neuron.2011.07.029

[35] Casas-Torremocha, D., Clascá, F. and Núñez, Á. (2017) Posterior Thalamic Nucleus Modulation of Tactile Stimuli Processing in Rat Motor and Primary Somatosensory Cortices. Frontiers in Neural Circuits, 11, 69. https://doi.org/10.3389/fncir.2017.00069

[36] Bopp, R., Holler-Rickauer, S., Martin, K.A. and Schuhknecht, G.F. (2017) An Ultrastructural Study of the Thalamic Input to Layer 4 of Primary Motor and Primary Somatosensory Cortex in the Mouse. Journal of Neuroscience, 37, 2435-2448. https://doi.org/10.1523/JNEUROSCI.2557-16.2017

[37] Friedrich, J., Mückschel, M. and Beste, C. (2018) Specific Properties of the SI and SII Somatosensory Areas and Their Effects on Motor Control: A System Neurophysiological Study. Brain Structure and Function, 223, 687-699. https://doi.org/10.1007/s00429-017-1515-y

[38] Thompson, A., Murphy, D., Dell'Acqua, F., Ecker, C., McAlonan, G., Howells, H., Baron-Cohen, S., Lai, M.C., Lombardo, M.V. (2017) Impaired Communication between the Motor and Somatosensory Homunculus Is Associated with Poor Manual Dexterity in Autism Spectrum Disorder. Biological Psychiatry, 81, 211-219. https://doi.org/10.1016/j.biopsych.2016.06.020

[39] Vanni, M.P. and Murphy, T.H. (2014) Mesoscale Transcranial Spontaneous Activity Mapping in GCaMP3 Transgenic Mice Reveals Extensive Reciprocal Connections between Areas of Somatomotor Cortex. Journal of Neuroscience, 34, 15931-15946. https://doi.org/10.1523/JNEUROSCI.1818-14.2014

[40] Rocco-Donovan, M., Ramos, R.L., Giraldo, S. and Brumberg, J.C. (2011) Characteristics of Synaptic Connections between Rodent Primary Somatosensory and Motor Cortices. Somatosensory \& Motor Research, 28, 63-72. https://doi.org/10.3109/08990220.2011.606660

[41] Krumova, E.K., Geber, C., Westermann, A. and Maier, C. (2012) Neuropathic Pain: Is Quantitative Sensory Testing Helpful? Current Diabetes Reports, 12, 393-402. https://doi.org/10.1007/s11892-012-0282-7

[42] Porto, F.H.G., Porto, G.C.L.M. and Brotto, M.W.L. (2016) Additional Tests to Investigate Neuropathic Pain. The Value of Electroneuromyography for Neuropathic Pain. Revista Dor, 17, 23-26. https://doi.org/10.5935/1806-0013.20160042 


\section{Highlights}

- The adapted CCI method by one loose ligature of the ischiadicus nervus causes neuropathic pain;

- Cold allodynia was found after 21 days of adapted CCI surgery;

- Acetone test is appropriate to evaluate the cold allodynia;

- Motor cortex stimulation attenuates the cold allodynia in CCI rats;

- $\mathrm{M}_{1}$-stimulation causes antinociception in rats with neuropathic pain. 